Research Paper

\title{
Upregulated SLC22A3 has a potential for improving survival of patients with head and neck squamous cell carcinoma receiving cisplatin treatment
}

\author{
Cheng-Ming Hsu ${ }^{1,2,3}$, Pai-Mei Lin ${ }^{4}$, Jan-Gowth Chang ${ }^{5,6,7}$, Hsin-Ching Lin', Shau- \\ Hsuan Li ${ }^{8}$, Sheng-Fung Lin ${ }^{9,10}$ and Ming-Yu Yang ${ }^{2,3}$ \\ ${ }^{1}$ Department of Otolaryngology, Chiayi Chang Gung Memorial Hospital and Chang Gung University College of Medicine, \\ Chiayi, Taiwan \\ ${ }^{2}$ Department of Otolaryngology, Kaohsiung Chang Gung Memorial Hospital and Chang Gung University College of Medicine, \\ Kaohsiung, Taiwan \\ ${ }^{3}$ Graduate Institute of Clinical Medical Sciences, College of Medicine, Chang Gung University, Tao-Yuan, Taiwan \\ ${ }^{4}$ Department of Nursing, I-Shou University, Kaohsiung, Taiwan \\ ${ }^{5}$ Department of Laboratory Medicine, China Medical University Hospital, Taichung, Taiwan \\ ${ }^{6}$ College of Medicine, China Medical University, Taichung, Taiwan \\ ${ }^{7}$ Epigenome Research Center, China Medical University Hospital, Taichung, Taiwan \\ ${ }^{8}$ Division of Hematology-Oncology, Department of Internal Medicine, Kaohsiung Chang Gung Memorial Hospital and Chang \\ Gung University College of Medicine, Kaohsiung, Taiwan \\ ${ }^{9}$ Division of Hematology-Oncology, Department of Internal Medicine, Kaohsiung Medical University Hospital, Kaohsiung, \\ Taiwan \\ ${ }^{10}$ Faculty of Medicine, Kaohsiung Medical University, Kaohsiung, Taiwan \\ Correspondence to: Ming-Yu Yang, email: yangmy@mail.cgu.edu.tw \\ Sheng-Fung Lin, email: shlin@cc.kmu.edu.tw
}

Keywords: SLC22A3, cancer therapy, cisplatin, survival, head and neck squamous cell carcinoma

Received: June 16, 2017 Accepted: August 17, $2017 \quad$ Published: September 04, 2017

Copyright: Hsu et al. This is an open-access article distributed under the terms of the Creative Commons Attribution License 3.0 (CC BY

3.0), which permits unrestricted use, distribution, and reproduction in any medium, provided the original author and source are credited.

\section{ABSTRACT}

Solute carrier family 22 member 3 (SLC22A3), also called organic cation transporter 3 (OCT3), is responsible for organic cation transport, which can eliminate many endogenous small organic cations, drugs, and toxins. This study investigated whether SLC22A3 expression is related to cisplatin uptake and the survival of patients with head and neck squamous cell carcinoma (HNSCC). Using immunohistochemical staining and digital image analysis, SLC22A3 expression was examined in 42 HNSCC patients who were postoperatively treated with or without adjuvant chemotherapy. SLC22A3-overexpressing SCC-4 cells and SLC22A3-knocked down SCC-25 cells were used to investigate the function of SLC22A3 in cisplatin uptake. We found that patients with higher SLC22A3 expression had longer survival times than those with lower SLC22A3 expression $(p=0.051)$. Moreover, among advanced T-stage patients receiving adjuvant cisplatin therapy, those with higher SLC22A3 expression had longer survival times than those with lower SLC22A3 expression $(p=0.006)$. An in vitro study demonstrated that SCC-25 cells with upregulated SLC22A3 expression were more sensitive to cisplatin than were SCC-4 cells with downregulated SLC22A3 expression. An increased uptake of cisplatin and an enhanced cytotoxic effect were observed in SLC22A3-overexpressing SCC-4 cells, and decreased uptake was found in SLC22A3-knocked down SCC-25 cells. Our results demonstrated that upregulated SLC22A3 expression can increase the cisplatin uptake and subsequently improve the survival of patients with HNSCC. 


\section{INTRODUCTION}

Head and neck squamous cell carcinoma (HNSCC) is the tenth most common cancer in men worldwide and is currently the seventh most common cause of cancerrelated death [1]. The standard treatments for HNSCC consist of surgery, radiotherapy, chemotherapy and combinations of these modalities [2]. Despite aggressive combination treatments, little progress has been made toward improving outcomes [3]. In its early stage, HNSCC may be curable with surgery, radiation, and chemotherapy. However, in the advanced stage, tumor recurrence and metastasis may occur after primary treatment and are associated with a poor outcome.

Solute carrier family 22 member 3 (SLC22A3), also called organic cation transporter 3 (OCT3), is an imprinted gene located on human chromosome 6 . The promoter of $S L C 22 A 3$ is located $155 \mathrm{~kb}$ from the $3^{\prime}$ end of the IGF2R gene, which is closely associated with $S L C 22 A 2 / O C T 2$ and SLC22A1/OCT1 [4]. SLC transporters (e.g. OCT2) are renal membrane transporters regarding its role in cisplatin nephrotoxicity. In mouse models of cisplatin ototoxicity, SLC transporters (e.g. CTR1 and OCT2) had been associated with cisplatin-induced ototoxicity. Organic cation/carnitine transporters rOctn1 and rOctn2 may mediate oxaliplatin induced neurotoxicity in rats [5]. SLC transporters expressed in the small intestine, liver, and kidney may play an important role in the disposition of cancer drugs [6] and expressed in cancer cells play an important role in the cellular uptake of anticancer drugs, which may be a determinant step of anticancer drug efficacy [6].

The role of SLC22A3/OCTs in metabolism is the uptake, intracellular inactivation, and biliary or urinary excretion of a broad spectrum of endogenous (e.g., catecholamines) and exogenous substrates (e.g., metformin and betablockers) and anticancer drugs (e.g., platin derivatives) [7-12]. Therefore, SLC22A3/OCTS may be associated with HNSCC and its prognosis.

Platinum agents are widely used in the treatment of cancer. Cisplatin (cis-diamminedichloroplatinum II, CDDP) was the first platinum agent to be synthesized, and it has since played an essential role in cancer chemotherapy for 30 years. However, cisplatin efficacy is far from optimal because of its poor tumor cell specificity and its hydrophilic properties, which limit the intracellular uptake of cisplatin. Therefore, developing strategies to improve its intracellular uptake may improve cisplatin efficacy and patient survival.

We hypothesized that the expression level of $S L C 22 A 3$ influences the anticancer effect of cisplatin against HNSCC. Therefore, in this study, we elucidated the effect of $S L C 22 A 3$ expression on cisplatin uptake by HNSCC cells with overexpression or knockdown of $S L C 22 A 3$. The survival was assessed to evaluate the response to chemotherapy of patients and to determine the correlation between the response and their expression levels of $S L C 22 A 3$.

\section{RESULTS}

\section{Patients with higher SLC22A3 expression had an improved survival rate after cisplatin therapy}

In this study, we investigated SLC22A3 protein expression in patients with HNSCC by using IHC staining. Table 1 lists the clinical pathological characteristics of the 42 patients enrolled in this study. Figure 1 shows IHC staining for different expression levels of SLC22A3 and the corresponding scores. Furthermore, patients were divided into two groups according to the difference in the scores of tumorous and adjacent normal tissue. The higher SLC22A3 expression group was defined as a score difference of 1 or 2, which implies higher expression in tumorous tissue than in adjacent normal tissue. The lower SLC22A3 expression group was defined as a score difference of 0 , which implies the same level of SLC22A3 expression in tumorous and adjacent normal tissues. A total of 30 and 12 patients were included in the higher and lower expression group, respectively (Table 2). The 2 -year survival rate in the higher expression group was higher than that in the lower expression group $(80.0 \%$ vs. $50.0 \%, p=0.051$ ) (Figure 2A). According to the head and neck cancer guideline of our hospital, 22 high-risk patients received adjuvant therapies including 5 -fluorouracil (FU) combined with cisplatin $(n=15)$ or cisplatin only $(n=7)$. We found that among patients receiving cisplatin therapy, those with higher SLC22A3 expression had a higher 2-year survival rate than those with lower SLC22A3 expression $(p=0.036)$ (Figure $2 \mathrm{~B})$. Further analysis revealed that advanced T-stage patients (Figure $2 \mathrm{C}$ ) and advanced clinical stage patients (Figure 2D) with higher SLC22A3 expression also had improved survival after cisplatin therapy ( $p=0.006$ and $p=0.034$, respectively).

\section{Sensitivity to cisplatin was higher in SLC22A3 higher expressing SCC-25 cells than in SLC22A3 lower expressing SCC-4 cells}

We examined the expression level-dependent effect of $S L C 22 A 3$ on cisplatin-induced cytotoxicity. Typically, SCC-25 cells exhibit higher SLC22A3 expression than SCC-4 cells (Figure 3A). Through an MTT assay, we found that the $\mathrm{IC}_{50}$ of cisplatin for SCC-4 cells was approximately $25 \mu \mathrm{M}$ at 3 days after treatment (Figure. 3B). However, after SCC-25 cells were treated with $10 \mu \mathrm{M}$ cisplatin, cell growth decreased drastically at 3 days after treatment (Figure 3C).

\section{Cytotoxic effect of cisplatin was enhanced by SLC22A3 overexpression in SCC-4 cells}

To elucidate the effects of $S L C 22 A 3$ expression on cisplatin-induced cytotoxicity, we transiently overexpressed SLC22A3 in SCC-4 cells (Figure 4A) and 
knocked down $S L C 22 A 3$ in SCC-25 cells (Figure 4B). When SLC22A3-overexpressing SCC-4 cells were treated with 5 and $25 \mu \mathrm{M}$ cisplatin for 72 hours, cisplatininduced cytotoxicity was strongly enhanced by $S L C 22 A 3$ expression (Figure 4C). However, cisplatin-induced cytotoxicity did not differ between SCC-25 cells with or without SLC22A3 knockdown (Figure 4D).

\section{Transport of cisplatin}

To further clarify the effect of SLC22A3 on the uptake of cisplatin, we examined the uptake of cisplatin in SLC22A3-overexpressing SCC-4 cells and SLC22A3knocked down SCC-25 cells. We observed increased uptake of cisplatin in SLC22A3-overexpressing SCC-4 cells (Figure 5A). However, decreased uptake of cisplatin was observed in SLC22A3-knocked down SCC-25 cells (Figure 5B).

\section{DISCUSSION}

SLC22A3 mRNA has been detected in the placenta, intestine, heart, brain, and kidney, but the distribution of $S L C 22 A 3$ in the plasma membrane and its physiological role are not yet clearly understood [13-15]. A recent study demonstrated that OCT1 and OCT3 play a role in the uptake of serotonin and histamine and metformin in the gastrointestinal tract [16].

$S L C 22 A 3$ has been extensively studied in many tissues and tumor cell lines and has been found to be associated with breast cancer, bladder cancer, and lung cancer [17-20]. However, only a few studies have investigated $S L C 22 A 3$ expression and its role in human malignancies including HNSCC.

In the present study, we graded the expression level of SLC22A3 according to IHC staining by using digital image analysis, and patients were divided into a higher SLC22A3 expression group (higher SLC22A3 expression in tumorous tissue than in normal tissue) and a lower SLC22A3 expression group (the same level of SLC22A3 expression in tumorous and normal tissues). We found a significant correlation between SLC22A3 expression and the response to adjuvant chemotherapy. Therefore, we hypothesized that the substrate specificity and expression level of SLC22A3 affect the anticancer effect of platinum agents against HNSCC. OCTs may play an important role in the treatment of malignant tumors because they are responsible for the cytotoxicity of platinum derivatives and are predictors of responses to small molecules [21]. Cisplatin plays an essential role in chemotherapy against solid tumors of the prostate, bladder, lung, and testis and HNSCC [22]. However, its efficacy is limited by its hydrophilic properties, which limit its intracellular uptake [23].

Cisplatin is activated when it enters the cell. It binds to the $\mathrm{N} 7$ reactive center on purine residues and can cause DNA damage in cancer cells, inhibiting cell division and resulting in apoptotic cell death [22]. The cisplatinDNA adduct level is the most critical determinant of the sensitivity of HNSCC cells to cisplatin [24].
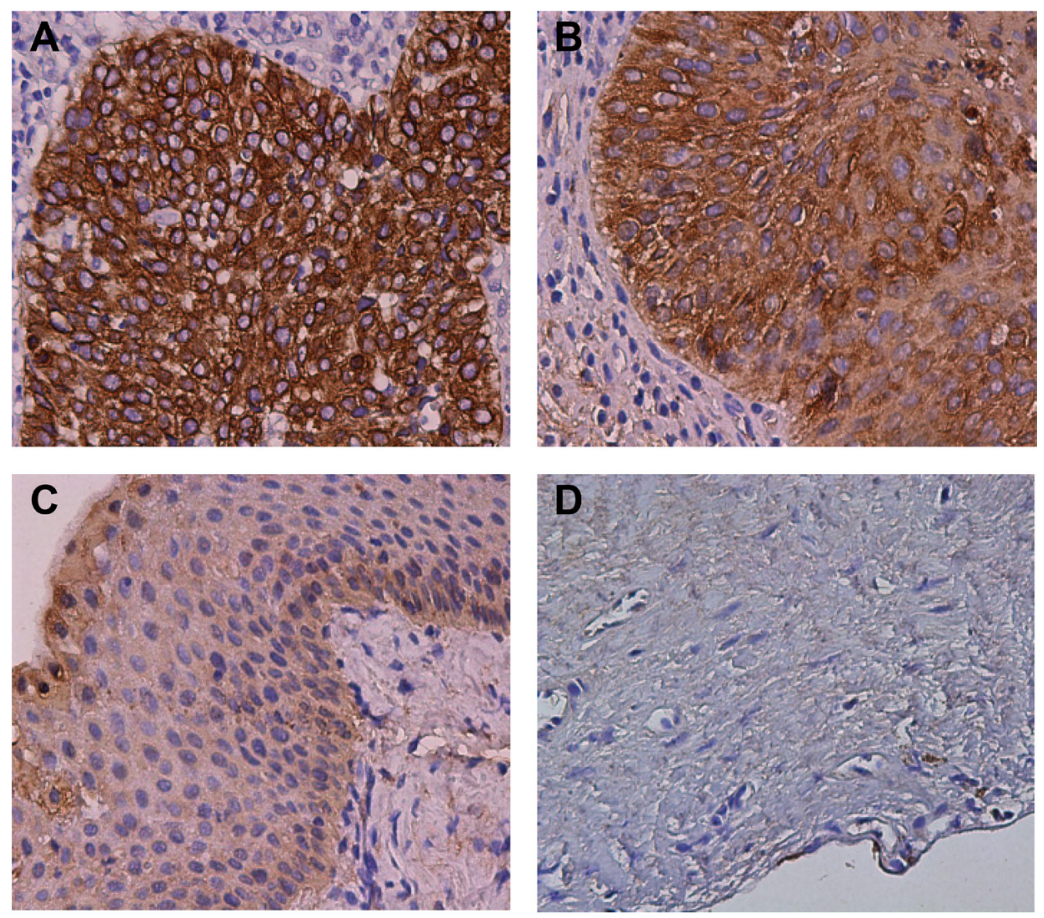

Figure 1: Immunohistochemical staining of SLC22A3 in HNSCC. Representative images of HNSCC show (A) high positive SLC22A3 expression (score $=+3$ ) and $($ B $)$ positive SLC22A3 expression (score $=+2$ ) in cancerous tissues and (C) low positive SLC22A3 expression (score $=+1)$ and (D) negative SLC22A3 expression (score $=0$ ) in adjacent noncancerous tissues. Original magnification: $\times 200$. 
Table 1: Characteristics of patients with HNSCC

\begin{tabular}{lc}
\hline Characteristic & No. of patients \\
\hline Sex & 40 \\
Male & 2 \\
Female & $53.7(30-76)$ \\
Median age, y (range) & \\
T stage & 5 \\
T1 & 13 \\
T2 & 6 \\
T3 & 16 \\
T4a & 1 \\
T4b & \\
N stage & 25 \\
N0 & 3 \\
N1 & 2 \\
N2a & 6 \\
N2b & 6 \\
N2c & \\
Primary tumor site & 26 \\
Oral cavity & 1 \\
Larynx & 2 \\
Supraglottic & 9 \\
Ororpharynx & 6 \\
Hypopharynx & \\
Tumor size & 20 \\
$<3$ cm & 22 \\
> 3 cm & \\
Neck metastasis & 17 \\
Positive & 25 \\
Negative & \\
Two-year survival & 30 \\
Expired & \\
Survived & \\
\hline & \\
& \\
\hline
\end{tabular}

Cisplatin is an important chemotherapeutic against solid tumors of the prostate, bladder, lung, testis, liver, and brain [25]. Concurrent treatment with high-dose cisplatin and radiotherapy represents the definitive adjuvant treatment for high-risk HNSCC [26]. However, the effect of cisplatin on recurrent HNSCC is weak. Passive transporters have been implicated to play a role in the cellular influx of cisplatin and carboplatin. The $S L C$ gene encodes a large family of passive transporters predominated by ion-coupled transporters and exchangers. These transporters include OCTs, which are highly expressed in the proximal tubules of the kidney and appear to be integral to development [15]. Recent studies have identified OCT as a membrane transporter capable of transporting cisplatin into cells. Therefore, a high level of SLC22A3 may benefit generating a favorable response to platin treatment. Similarly, a recent study showed that SLC22A3 expression in renal cell carcinoma cell lines also enhanced sensitivity to chemotherapeutics such as melphalan, irinotecan, and vincristine [27]. This finding may explain why the survival of patients in the higher expression group had improved after cisplatin treatment.

In this study, we found that the expression of SLC22A3 was low in SCC-4 cells and was high in SCC-25 cells. Sensitivity to cisplatin also differed between the two cell lines. SCC-25 cells with higher levels of SLC22A3 expression showed high sensitivity and strong cisplatininduced cytotoxicity, whereas SCC-4 cells with lower SLC22A3 expression showed low sensitivity and weak cisplatin-induced cytotoxicity.

In a functional study, we found that upregulated SLC22A3 expression in SCC-4 cells increased sensitivity to cisplatin and enhanced cisplatin-induced cytotoxicity. However, the knockdown of SLC22A3 in SCC-25 cells did not reduce sensitivity to cisplatin or cisplatin-induced cytotoxicity. This finding may be because SLC22A3 is not the only cisplatin transporter or channel. A recent study demonstrated that copper transporter 1 (CTR1) markedly influenced the uptake of all clinically used platinumcontaining drugs, suggesting that CTR1 also transports 
Table 2: Characteristics of patients with HNSCC stratified by SLC22A3 expression level

\begin{tabular}{lccc}
\hline Characteristic & $\begin{array}{c}\text { SLC22A3 } \\
\text { Higher expression }\end{array}$ & $\begin{array}{c}\text { SLC22A3 } \\
\text { Lower expression }\end{array}$ & $\boldsymbol{p}$ value \\
\hline No. of patients & $30(71.4 \%)$ & $12(28.6 \%)$ & \\
Gender & & & 0.999 \\
$\quad$ Male/Female & $28 / 2$ & $12 / 0$ & 0.135 \\
Median age, y (range) & $55.3(38-76)$ & $50.0(30-70)$ & \\
T Stage & & & 0.999 \\
$\quad$ T1 + T2 & 14 & 5 & 0.426 \\
$\quad$ T3 + T4 & 16 & 7 & \\
Tumor size $>3$ cm & $11(36.7 \%)$ & $6(50.0 \%)$ & 0.052 \\
Two-year survival & & & \\
Survival/Expired & $24 / 6(80.0 \%)$ & $6 / 6(50.0 \%)$ & 0.499 \\
Treatment & & & \\
$\quad$ Cisplatin & $17(56.7 \%)$ & $5(41.7 \%)$ & \\
$\quad$ Non-cisplatin & $13(43.3 \%)$ & $7(58.3 \%)$ & \\
\hline
\end{tabular}

a) Higher expression: score difference $=1$ or 2

b) Lower expression: score difference $=0$

c) Data are expressed as the mean $\pm \mathrm{SD}$.
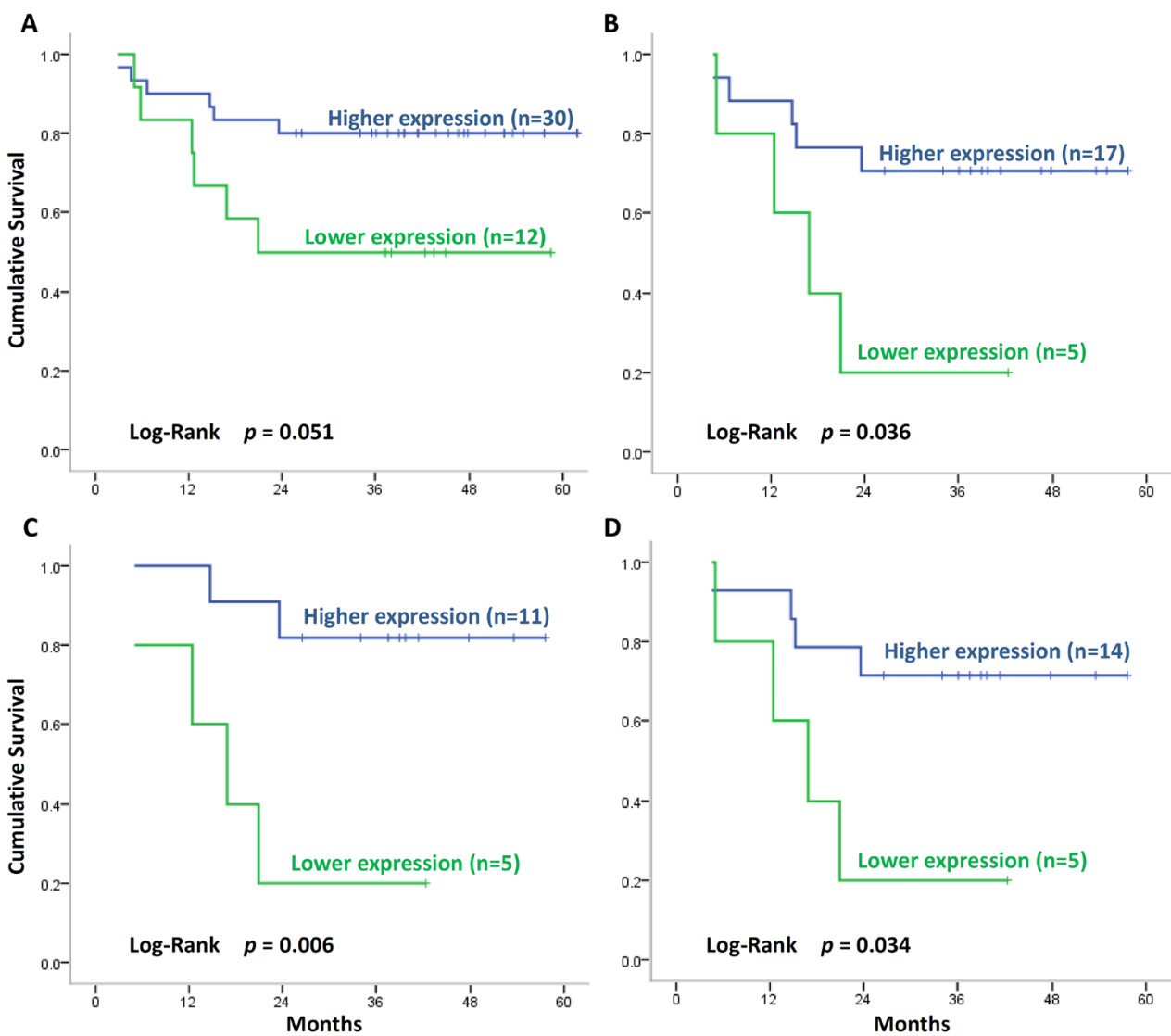

Figure 2: Survival of patients with HNSCC and SLC22A3 expression. (A) Survival of patients with higher SLC22A3 expression (score difference $=1$ or 2 ) and lower SLC22A3 expression (score difference $=0$ ). Patients with higher SLC22A3 expression exhibited improved survival. (B) Survival of patients receiving cisplatin therapy. Patients with higher SLC22A3 expression exhibited improved survival after cisplatin therapy. (C) Survival of advanced $T$-stage patients receiving cisplatin therapy. Advanced T-stage patients with higher SLC22A3 expression exhibited improved survival after cisplatin therapy. (D) Survival of advanced clinical stage patients receiving cisplatin therapy. Advanced clinical stage patients with higher SLC22A3 expression exhibited improved survival after cisplatin therapy. 
DDP $[28,29]$. Therefore, despite SLC22A3 knockdown, cisplatin may still enter the cells through CTR1.

In the uptake study, we found that SCC-4 cells with upregulated $S L C 22 A 3$ expression exhibited increased uptake of cisplatin, which may explain the accompanying increased sensitivity to cisplatin and enhanced cisplatininduced cytotoxicity. Hence, the efficacy of cisplatin treatment may be higher in patients with higher SLC22A3 expression, and these patients may subsequently exhibit improved survival. By contrast, SCC-25 cells with the knockdown of $S L C 22 A 3$ exhibited a reduced uptake of cisplatin. However, the reduced uptake of cisplatin by closing the SLC22A3 channel was insufficient to fully enhance resistance to cisplatin because the uptake of cisplatin may occur through the alternative transporter CTR1.

We speculate that when the expression of SLC22A3 is increased in recurrent or metastatic tumors, these tumors may become platinum-resistant. In our study, we enrolled only primary tumor and excluded the recurrent and metastatic tumors, therefore we have no direct evidence to know the changes of SLC22A3 expression occurring in recurrent tumors or metastatic tumors $v s$. primary tumors. It has been suggested that the development of acquired platinum resistance involved epithelial to mesenchymal transition (EMT), resulting in tumor aggressiveness with motile function of cancer cells [30]. Whether SLC22A3
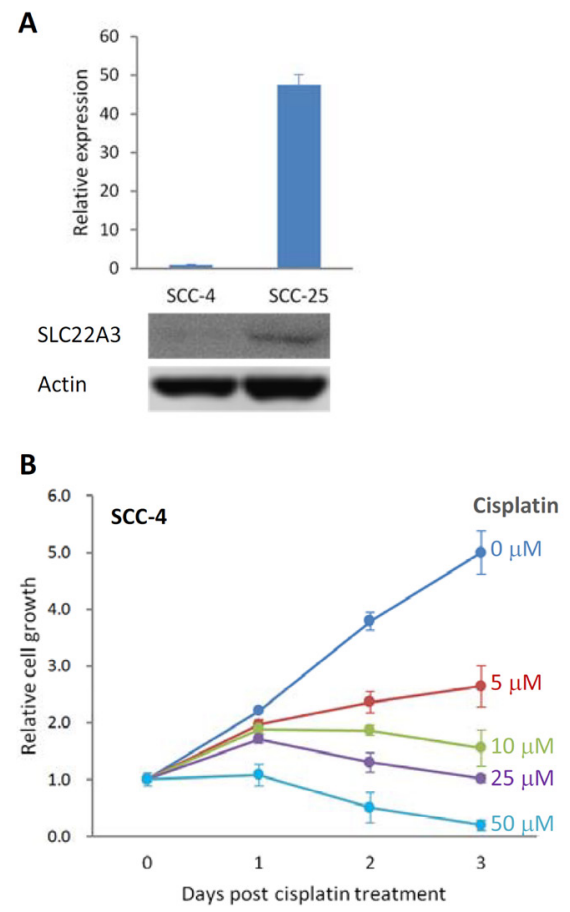

is also involved in the development of acquired platinum resistance will be the main issue for our next study to explore and we can start from comparing the differential SLC22A3 expression between recurrent tumor and primary tumor.

We hypothesized that higher $S L C 22 A 3$ expression in cancer cells may enhance the uptake of cisplatin after cisplatin treatment and, in turn, reduce the survival of cancer cells, and the opposite may hold true for cancer cells with lower SLC22A3 expression. Therefore, $S L C 22 A 3$ may be a novel therapeutic marker for patients with HNSCC receiving cisplatin-based chemotherapy. IHC staining of tumorous tissue samples by using SLC22A3 antibody and the quantification of the immunostained samples are also required to establish a parameter for clinical decision-making processes, particularly for patients requiring adjuvant treatment.

Our future goal is to establish screening methods for SLC22A3 expression in peripheral blood samples or biopsied tissues to predict the response to and outcome of cisplatin treatment. Imaging with radiolabeled cisplatin might have major associations with the treatment outcome. If this can be accomplished, we can identify the poor responders to cisplatin treatment and then select a more efficient therapy, such as targeted therapy or immunotherapy, for these individuals. We also hope to identify the activators of SLC22A3 expression to enhance

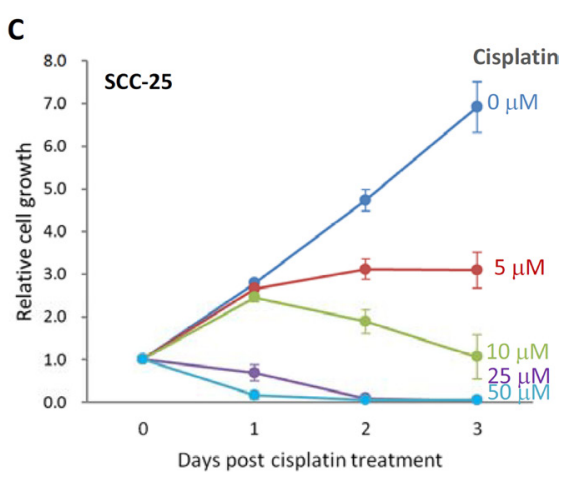

Figure 3: Sensitivity to cisplatin correlated with the level of SLC22A3 expression. (A) SLC22A3 expression was lower in SCC-4 cells but higher in SCC-25 cells, as determined by qRT-PCR (upper panel) and Western blotting (lower panel). The value of SLC22A3 expression in SCC-4 cells is designated 1, and the expression level in SCC-25 cells is related to this value. An MTT assay was performed to examine the effect of cell toxicity for SCC-4 cells (B) and SCC-25 cells (C) after treatment with various concentrations of cisplatin for 3 days. The relative cell growth is presented by comparing the data with day 0 . Data are presented as the mean and SE of three independent experiments. 
the uptake of cisplatin by cancer cells. However, as the effect of anticancer effects are enhanced by the upregulated SLC22A3, the side effects of chemotherapy may also be enhanced. So we need to be more careful in the future application.

In conclusion, the study findings suggest that cisplatin-induced cytotoxicity is mediated by the uptake of cisplatin by cancer cells through SLC22A3, and SLC22A3 expression in cancer cells is a potential biomarker for including cisplatin in cancer chemotherapy. Our findings reveal some strategies for improving the treatment outcome for advanced HNSCC.

A

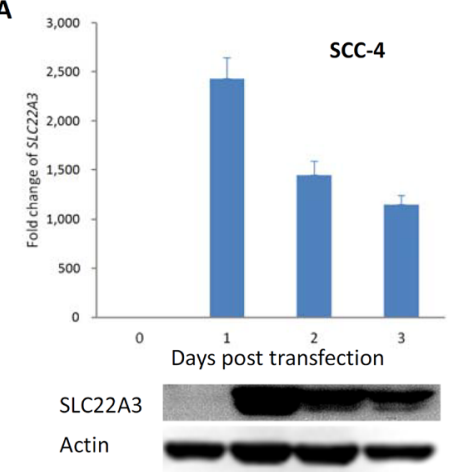

\section{MATERIALS AND METHODS}

\section{Patients and samples}

Cancerous tissues and adjacent noncancerous tissues were obtained from 42 patients diagnosed as having HNSCC who were undergoing surgery at the Department of Otolaryngology, Kaohsiung Chang Gung Memorial Hospital, Taiwan, between 2011 and 2013. Immediately after resection, the obtained specimens were snap-frozen in liquid nitrogen and stored at $-80^{\circ} \mathrm{C}$ until use. Prior to tissue acquisition, informed consent was obtained from

B

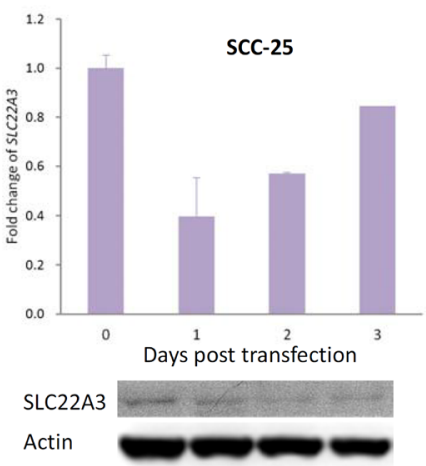

C
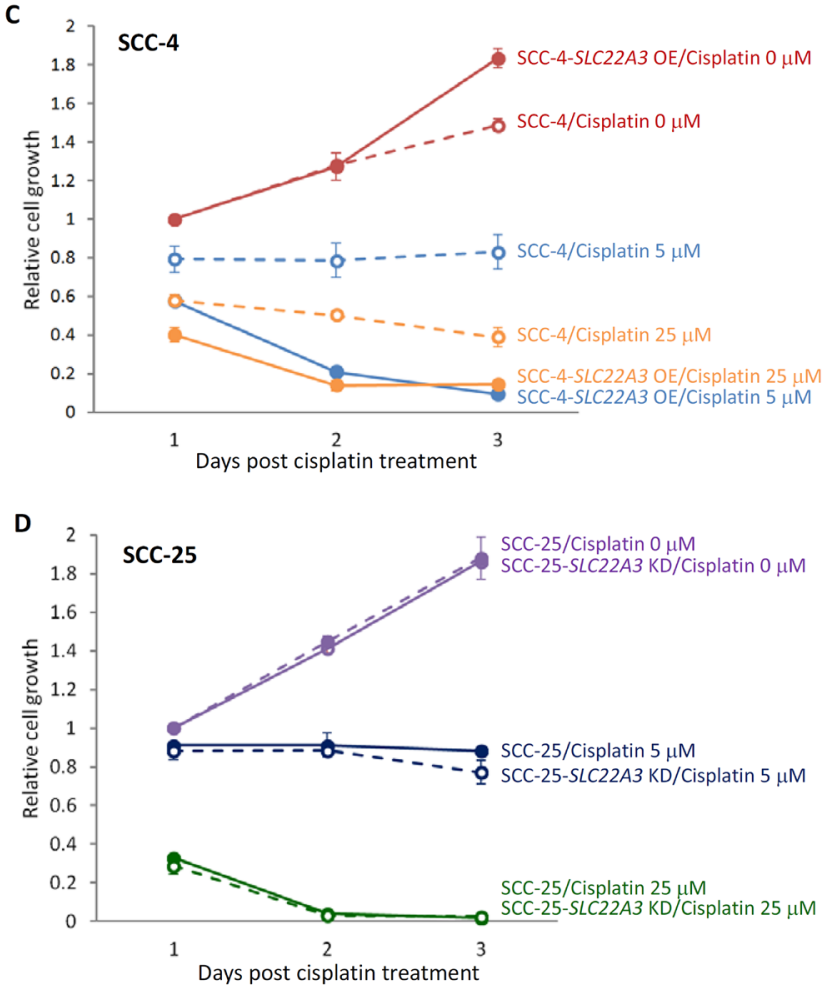

Figure 4: Cytotoxic effect of cisplatin was enhanced by SLC22A3 overexpression. (A) $S L C 22 A 3$ expression was upregulated in SCC-4 cells transfected with pCMV6-AC-MycDDK plasmid vector containing SLC22A3, as determined by qRT-PCR (upper panel) and Western blotting (lower panel). (B) SLC22A3 expression was downregulated in SCC-25 cells transfected with $S L C 22 A 3$ siRNA, as determined by qRT-PCR (upper panel) and Western blotting (lower panel). The value of $S L C 22 A 3$ expression on the day of transfection is designated 1 , and the expression levels on different days after transfection are related to this value. An MTT assay was performed to examine the the effect of cell toxicity for SCC-4 cells (C) and SCC-25 cells (D) at 3 days after transfection and treatment with 0, 5, and 25 $\mathrm{mM}$ cisplatin. Data are presented as the mean and SE of three independent experiments. 
all enrolled patients. This study was approved by the Institutional Review Board of Chang Gung Memorial Hospital (IRB Approval No. 100-4455A3).

\section{Disease severity and treatment of patients with HNSCC}

The TNM staging system was established by the American Joint Committee on Cancer and includes the tumor $(\mathrm{T})$, neck lymph node $(\mathrm{N})$, and metastasis $(\mathrm{M})$ status. Stages I-IV represent the general cancer status from mild (stage I) to severe (stage IV) and are closely related to prognosis and the therapy response. In the present study, we divided the enrolled patients into two disease severity groups for a correlation analysis of SLC22A3 expression: (1) early stage (stages I and II) and advanced stage (stages III and IV) groups, and (2) tumor stage T1-T2 and T3-T4 groups. Patients with HNSCC were treated in accordance with the head and neck cancer guidelines of the National Comprehensive Cancer Network: cisplatin at dosages of $60-75 \mathrm{mg} / \mathrm{m}^{2}$ by intravenous infusion for 4 hours with or without 5 -FU at dosages of $600-750 \mathrm{mg} / \mathrm{m}^{2}$ per 24 hours as a 96-hour continuous intravenous infusion, repeated every 3 weeks per cycle. The survival status of patients was followed up for at least 2 years after surgery. Overall survival was calculated from the date of diagnosis until death or last follow-up.

\section{Immunohistochemical (IHC) staining of SLC22A3}

IHC staining of cancerous tissues and adjacent noncancerous tissues from patients with HNSCC was performed. Tissue sections were incubated with monoclonal antibody against SLC22A3 (1:200 dilution; Epitomics, Burlingame, CA, USA) for 1 hour and then incubated with biotinylated goat anti-rabbit antibody for 30 minutes. Finally, the specific binding was visualized using a horseradish peroxidase-diaminobenzidine staining kit (Abcam Inc., MA, USA).

\section{Digital image analysis}

All IHC-stained slides were observed under a light microscope (Zeiss, Gottingen, Germany) at $\times 400$ magnification. Four 2-dimensional images were taken
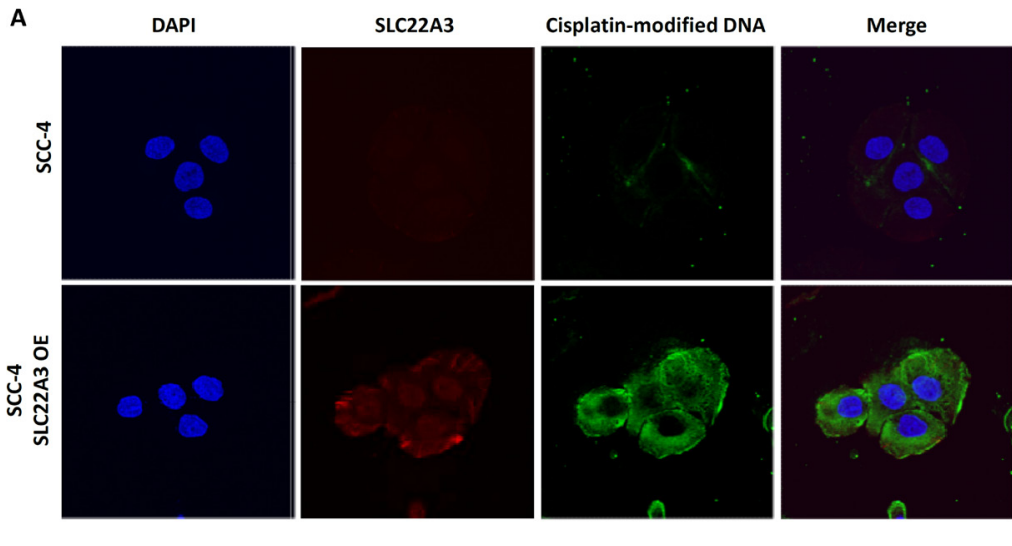

B

DAPI

SLC22A3

Cisplatin-modified DNA

Merge
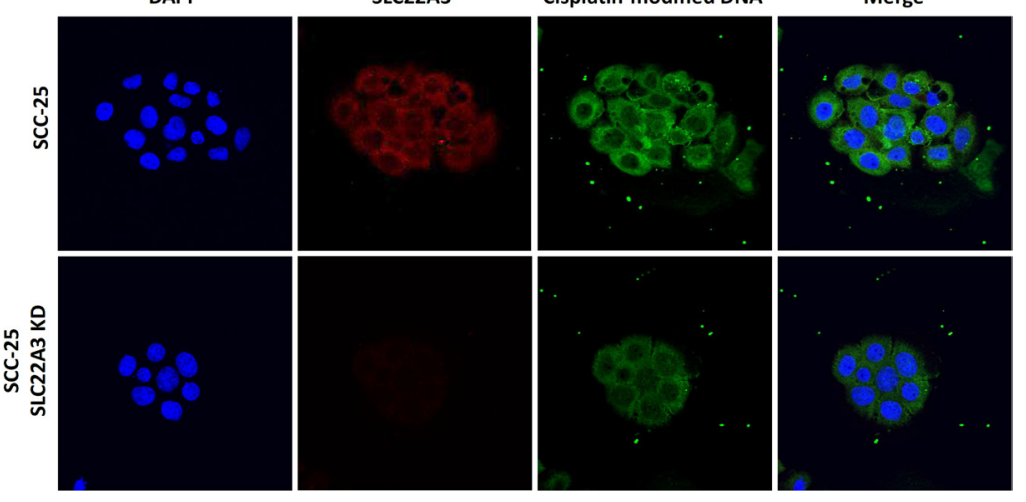

Figure 5: Uptake of cisplatin by SCC-4 and SCC-25 cells. (A) SCC-4 and SLC22A3 (OCT)-overexpressing SCC-4 cells and (B) SCC-25 and SLC22A3 (OCT)-knocked down SCC-25 cells were treated with $50 \mu \mathrm{M}$ cisplatin for 6 hours. Confocal microscopy was performed using anti-cisplatin-modified DNA antibody (primary antibody) plus TRITC-labelled goat anti-rabbit IgG (secondary antibody) and anti-SLC22A3 (OCT) antibody (primary antibody) plus Alexa Fluor 488-labelled goat anti-rat IgG (secondary antibody). DNA was counterstained with DAPI. The red signal represents SLC22A3 protein and the blue signal represents nucleus. The green signal represents influx of cisplatin-binding DNA. 
for each slide by using a microscope camera (Zeiss). To assess SLC22A3 expression, the percentage of area and integrated density (staining intensity) were calculated using ImageJ free software, version 1.410 (NIH, Maryland, USA). The relative intensity of IHC staining was quantified using ImageJ64 and was normalized to that of an internal control. According to the standard grading procedure, staining intensity was assigned scores using a 4-tier scoring system; that is, 3+, high positive; $2+$, positive; $1+$, low positive; and 0 , negative. Subsequently, patients were divided into three groups according to the difference in the scores assigned to tumorous and normal tissues; that is, score differences of 2,1 , and 0 , in a blinded manner.

\section{Cell cultures}

Two human HNSCC cell lines, SCC-4 and SCC25 (both tongue squamous cell carcinoma cell lines), used in this study were purchased from Food Industry Research and Development Institute, Taiwan. These cells were maintained in MEM (Minimum Essential Medium Eagle)-F12 medium (Invitrogen, Carlsbad, CA, USA) containing $0.4 \mu \mathrm{g} / \mathrm{mL}$ hydrocortisone (Sigma-Aldrich, St. Louis, MO, USA) and $10 \% \mathrm{FBS}$ and were grown at $37^{\circ} \mathrm{C}$ with $5 \% \mathrm{CO}_{2}$.

\section{MTT assay}

SCC-4 and SCC-25 cells were treated with various concentrations of cisplatin or PBS (Phosphate-Buffered Saline, as control); subsequently, the percentages of metabolically active cells were determined on the basis of the mitochondrial conversion of MTT into formazine. In brief, after cells were treated with or without cisplatin for different incubation times, culture media were replaced with DMEM/F-12 (Dulbecco's Modified Eagle's Medium, without phenol) containing 0.02\% MTT (Sigma-Aldrich) and incubated for 4 hours; subsequently, the medium was replaced with $200 \mu \mathrm{L}$ of dimethyl sulfoxide per well. The results were assessed in a 96-well format plate reader by measuring the absorbance at a wavelength of $595 \mathrm{~nm}$ on a DTX880 Multimode Detector (Beckman Coulter, Brea, CA, USA).

\section{Transfection}

For the transient expression and knockdown of $S L C 22 A 3$, pCMV6-AC-MycDDK plasmid vector DNA containing SLC22A3 (OriGene Technologies, Rockville, MD, USA) and SLC22A3 Stealth siRNA (ThermoFisher Scientific, NY, USA) were used, respectively. Cells (4000 cells/well) were transfected with $3 \mu \mathrm{g}$ of SLC22A3 overexpression plasmid DNA or SLC22A3 siRNA per well by using $1 \mu \mathrm{L}$ of Lipofectamine 2000 (Invitrogen), according to the manufacturer's instructions. After transfection for 30 minutes, cells were treated with various concentrations of cisplatin $(0,5,10,25$, and $50 \mu \mathrm{M})$ and were harvested on days 1,2 , and 3 after transfection.

\section{Cisplatin uptake and immunofluorescence staining}

SCC-4 and SCC-25 cells were transfected with SLC22A3-overexpressing plasmids and SLC22A3 siRNA, respectively. On the next day, cells were cultured for 6 hours in a medium containing $50 \mu \mathrm{M}$ cisplatin. Cells were then fixed with $4 \%$ paraformaldehyde, penetrated by freezing at $-80^{\circ} \mathrm{C}$, and permeated with $0.5 \%$ Triton $\mathrm{X}-100$ in PBS. After cells were blocked with $1 \%$ bovine serum albumin, they were stained with rat anti-cisplatin modified DNA antibody [CP9/19] (1:100 dilution; Abcam) for 16 hours at room temperature and were then stained with rabbit anti-SLC22A3 antibody (1:100 dilution; Abcam) for 4 hours. The secondary antibodies of goat anti-rabbit IgG labeled with TRITC (Sigma-Aldrich) and goat anti-rat IgG labeled with Alexa Fluor 488 (Abcam) were added at 1:400 dilution and incubated at room temperature for 1 hour. Slides were mounted with a mounting solution containing DAPI (4',6-Diamidino-2-Phenylindole, Dilactate) (Vector Lab, Burlingame, CA, USA) and were then analyzed using a confocal microscope (Fluoview FV10i; Olympus, Tokyo, Japan).

\section{Statistical analyses}

Kaplan-Meier survival curves were compared using a log-rank test. The Pearson chi-square test was used to determine the differences among categorical variables, and the Student $t$ test and ANOVA were used to determine the differences among continuous variables. All tests were two-sided, and results with $p<0.05$ were considered statistically significant. All statistical analyses were performed using SPSS version 13.0 for Windows (SPSS, Chicago, IL, USA).

\section{CONFLICTS OF INTEREST}

None declared.

\section{GRANT SUPPORT}

This work was supported in part by grants from Chang Gung Memorial Hospital (grant numbers CMRPG8B0361, CMRPG8B0362, CMRPG8B0363, CMRPG6F0511, CMRPD8E0171, CMRPD8D0292, CMRPD8F0761); Kaohsiung Medical University Hospital (grant numbers KMUH102-2T03, KMUH104-4R12); and the Ministry of Science and Technology of Taiwan (grant numbers MOST 100-2314-B-182A-023, MOST 101-2314-B-182A-051, MOST 103-2314-B-182A-063). 


\section{REFERENCES}

1. Mehanna H, Paleri V, West CM, Nutting C. Head and neck cancer-part 1: epidemiology, presentation, and preservation. Clin Otolaryngol. 2011; 36:65-8.

2. Dronkers EA, Mes SW, Wieringa MH, van der Schroeff MP, Baatenburg de Jong RJ. Noncompliance to guidelines in head and neck cancer treatment; associated factors for both patient and physician. BMC Cancer. 2015; 15:515.

3. Massano J, Regateiro FS, Januário G, Ferreira A. Oral squamous cell carcinoma: review of prognostic and predictive factors. Oral Surg Oral Med Oral Pathol Oral Radiol Endod. 2006; 102:67-76.

4. Verhaagh S, Schweifer N, Barlow DP, Zwart R. Cloning of the mouse and human solute carrier 22a3 (Slc22a3/ SLC22A3) identifies a conserved cluster of three organic cation transporters on mouse chromosome 17 and human 6q26-q27. Genomics. 1999; 55:209-18.

5. Liu JJ, Lu J, McKeage MJ. Membrane transporters as determinants of the pharmacology of platinum anticancer drugs. Curr Cancer Drug Targets. 2012; 12:962-86.

6. Li Q, Shu Y. Role of solute carriers in response to anticancer drugs. Mol Cell Ther. 2014; 2:15.

7. Nies AT, Koepsell H, Winter S, Burk O, Klein K, Kerb R, Zanger UM, Keppler D, Schwab M, Schaeffeler E. Expression of organic cation transporters OCT1 (SLC22A1) and OCT3 (SLC22A3) is affected by genetic factors and cholestasis in human liver. Hepatology. 2009; 50:1227-40.

8. Sakata T, Anzai N, Kimura T, Miura D, Fukutomi T, Takeda M, Sakurai H, Endou H. Functional analysis of human organic cation transporter OCT3 (SLC22A3) polymorphisms. J Pharmacol Sci. 2010; 113:263-6.

9. Ahmadimoghaddam D, Hofman J, Zemankova L, Nachtigal P, Dolezelova E, Cerveny L, Ceckova M, Micuda S, Staud F. Synchronized activity of organic cation transporter 3 (Oct3/Slc22a3) and multidrug and toxin extrusion 1 (Mate1/ Slc47a1) transporter in transplacental passage of MPP+ in rat. Toxicol Sci. 2012; 128:471-81.

10. Heise M, Lautem A, Knapstein J, Schattenberg JM, HoppeLotichius M, Foltys D, Weiler N, Zimmermann A, Schad A, Gründemann D, Otto G, Galle PR, Schuchmann M, et al. Downregulation of organic cation transporters OCT1 (SLC22A1) and OCT3 (SLC22A3) in human hepatocellular carcinoma and their prognostic significance. BMC Cancer. 2012; 12:109.

11. Ahmadimoghaddam D, Staud F. Transfer of metformin across the rat placenta is mediated by organic cation transporter 3 (OCT3/SLC22A3) and multidrug and toxin extrusion 1 (MATE1/SLC47A1) protein. Reprod Toxicol. 2013; 39:17-22.

12. Ahmadimoghaddam D, Zemankova L, Nachtigal $P$, Dolezelova E, Neumanova Z, Cerveny L, Ceckova M, Kacerovský M, Micuda S, Staud F. Organic cation transporter 3 (OCT3/SLC22A3) and multidrug and toxin extrusion 1 (MATE1/SLC47A1) transporter in the placenta and fetal tissues: expression profile and fetus protective role at different stages of gestation. Biol Reprod. 2013; 88:55.

13. Wu X, Kekuda R, Huang W, Fei YJ, Leibach FH, Chen J, Conway SJ, Ganapathy V. Identity of the organic cation transporter OCT3 as the extraneuronal monoamine transporter (uptake2) and evidence for the expression of the transporter in the brain. J Biol Chem. 1998; 273:32776-86.

14. Kekuda R, Prasad PD, Wu X, Wang H, Fei YJ, Leibach FH, Ganapathy V. Cloning and functional characterization of a potential-sensitive, polyspecific organic cation transporter (OCT3) most abundantly expressed in placenta. J Biol Chem. 1998; 273:15971-9.

15. Wu X, Huang W, Ganapathy ME, Wang H, Kekuda R, Conway SJ, Leibach FH, Ganapathy V. Structure, function, and regional distribution of the organic cation transporter OCT3 in the kidney. Am J Physiol Renal Physiol. 2000; 279:F449-58.

16. Yee SW, Lin L, Merski M, Keiser MJ, Gupta A, Zhang Y, Chien HC, Shoichet BK, Giacomini KM. Prediction and validation of enzyme and transporter off-targets for metformin. J Pharmacokinet Pharmacodyn. 2015; 42:463-75.

17. Wang P, Branch DR, Bali M, Schultz GA, Goss PE, Jin T. The POU homeodomain protein OCT3 as a potential transcriptional activator for fibroblast growth factor-4 (FGF-4) in human breast cancer cells. Biochem J. 2003; 375:199-205.

18. Ma N, Thanan R, Kobayashi H, Hammam O, Wishahi M, El Leithy T, Hiraku Y, Amro K, Oikawa S, Ohnishi S, Murata M, Kawanishi S. Nitrative DNA damage and Oct3/4 expression in urinary bladder cancer with Schistosoma haematobium infection. Biochem Biophys Res Commun. 2011; 414:344-9.

19. Iida H, Suzuki M, Goitsuka R, Ueno H. Hypoxia induces CD133 expression in human lung cancer cells by upregulation of OCT3/4 and SOX2. Int J Oncol. 2012; 40:71-9.

20. Ling GQ, Chen DB, Wang BQ, Zhang LS. Expression of the pluripotency markers Oct3/4, Nanog and Sox2 in human breast cancer cell lines. Oncol Lett. 2012; 4:1264-8.

21. Li Q, Peng X, Yang H, Rodriguez JA, Shu Y. Contribution of organic cation transporter 3 to cisplatin cytotoxicity in human cervical cancer cells. J Pharm Sci. 2012; 101:394404.

22. Kuo MT, Chen HH, Song IS, Savaraj N, Ishikawa T. The roles of copper transporters in cisplatin resistance. Cancer Metastasis Rev. 2007; 26:71-83.

23. Dasari S, Tchounwou PB. Cisplatin in cancer therapy: molecular mechanisms of action. Eur J Pharmacol. 2014; 740:364-78.

24. Martens-de Kemp SR, Dalm SU, Wijnolts FM, Brink A, Honeywell RJ, Peters GJ, Braakhuis BJ, Brakenhoff RH. DNA-bound platinum is the major determinant of cisplatin sensitivity in head and neck squamous carcinoma cells. PLoS One. 2013; 8:e61555. 
25. Ho GY, Woodward N, Coward JI. Cisplatin versus carboplatin: comparative review of therapeutic management in solid malignancies. Crit Rev Oncol Hematol. 2016; 102:37-46.

26. Seiwert TY, Salama JK, Vokes EE. The chemoradiation paradigm in head and neck cancer. Nat Clin Pract Oncol. 2007; 4:156-71.

27. Shnitsar V, Eckardt R, Gupta S, Grottker J, Müller GA, Koepsell H, Burckhardt G, Hagos Y. Expression of human organic cation transporter 3 in kidney carcinoma cell lines increases chemosensitivity to melphalan, irinotecan, and vincristine. Cancer Res. 2009; 69:1494-501.

28. Lin X, Okuda T, Holzer A, Howell SB. The copper transporter CTR1 regulates cisplatin uptake in Saccharomyces cerevisiae. Mol Pharmacol. 2002; 62:1154-9.
29. Ishida S, Lee J, Thiele DJ, Herskowitz I. Uptake of the anticancer drug cisplatin mediated by the copper transporter Ctr1 in yeast and mammals. Proc Natl Acad Sci USA. 2002; 99:14298-302.

30. Tanaka N, Kosaka T, Miyazaki Y, Mikami S, Niwa N, Otsuka Y, Minamishima YA, Mizuno R, Kikuchi E, Miyajima A, Sabe H, Okada Y, Uhlén P, et al. Acquired platinum resistance involves epithelial to mesenchymal transition through ubiquitin ligase FBXO32 dysregulation. JCI Insight. 2016; 1:e83654. 